\title{
Extraordinary disease-free survival in a rare malignant extrarenal rhabdoid tumor: a case report and review of the literature
}

\author{
Francesco D'Amico 1,2,6* ${ }^{*}$, Alessandra Bertacco ${ }^{1}$, Maurizio Cesari ${ }^{3}$, Claudia Mescoli ${ }^{4}$, Giorgio Caturegli², \\ Gabriel Gondolesi ${ }^{5}$ and Umberto Cillo ${ }^{1}$
}

\begin{abstract}
Background: Malignant extrarenal rhabdoid tumor of the gastrointestinal tract is rarely reported in the literature. It is characterized by poor prognosis and aggressive metastatic features.

A literature review evidenced only 19 cases, with poor outcome.

Case presentation: We report a case of a colonic "pure" malignant extrarenal rhabdoid tumor with metastatic nodes in a 65-year-old Caucasian man. He was treated surgically with no recurrence, no adjuvant chemotherapy, and with 4-year survival without disease at the time of the submission of this article.

Conclusions: We present an extraordinary case of long-term survival due to the extended surgical treatment. We believe that the absence of organ metastasis at presentation is a positive prognostic factor, although pathology confirmed node involvement (13/38 positive) on microscopy.
\end{abstract}

Keywords: Extrarenal rhabdoid tumor, Rare colon tumor, MERT, Extended surgery, Literature review, Case report

\section{Background}

A malignant rhabdoid tumor (MRT) is a subtype of Wilms' tumor; first described by Beckwith and Palmer in 1978 [1]. It is most common in children, usually with a renal localization. Adult forms are rare, and they are characterized by a poor prognosis. However, similar lesions arising in soft tissue and other sites have been reported and referred to as malignant extrarenal rhabdoid tumors (MERTs), which have been accepted as a clinical-pathological entity of their own. They share common histology and immunophenotype, and can be mixed with different types of neoplasms (carcinomas, melanomas, and sarcomas). The extrarenal variants are very aggressive, and have most often been located in the central nervous system (CNS), liver, soft tissue, and colon [2]. Presentation in the gastrointestinal (GI) tract is rare and the prognosis of the affected patient is poor

\footnotetext{
* Correspondence: drdamico@hotmail.com

${ }^{1}$ Department of Surgery, Oncology and Gastroenterology, Hepatobiliary

Surgery and Liver Transplantation, Padova University, Padova, Italy

${ }^{2}$ Department of Surgery, Division of Transplantation and Immunology, Yale

University, New Haven, CT, USA

Full list of author information is available at the end of the article
}

due to the aggressive nature of this disease. MRT is characterized by early diffuse metastasis, with death generally occurring within 6 months from initial diagnosis [3].

\section{Case presentation}

A 65-year-old Caucasian man was admitted to Padova University Hospital for diffuse abdominal pain associated with generalized weakness, decreased oral intake, and weight loss of $20 \mathrm{~kg}$ in 2 months.

A physical examination at admission showed pale conjunctiva and skin, bilateral auscultation revealed clear lungs, and heart auscultation did not elicit any murmurs, rubs, or gallop.

An abdominal examination induced abdominal pain with rebound tenderness; bowel sounds were present in all quadrants. A right inguinal mass suggestive of lymphadenopathy was found on examination. The rest of the physical examination was unremarkable.

Patient history revealed ischemic cardiomyopathy treated with endovascular coronary revascularization, diabetes, and depression. Diverticulosis of the colon and sigmoid polyposis were reported in a colonoscopy 
performed the previous year. No family history of malignancy was reported. During the recovery, our patient underwent esophagogastroduodenoscopy (EGD), which was negative, and a colonoscopy, which showed an ab-extrinsic compression of the cecum.

A total body computed tomography (CT) scan was performed, and it described a $10 \times 8 \times 10 \mathrm{~cm}$ mass invading the cecum and ascending colon (Fig. 1a) with lymphadenopathy. No metastasis was found. Tumor markers were negative.

Our patient underwent an elective right hemicolectomy with extended lymphadenectomy in the hepatogastric and aortocaval areas, and renal fat excision. The post-operative period was uneventful and he was discharged from the surgical unit on post-operative day 6 .

The pathologic diagnosis was rhabdoid tumor. The tumor stage was defined as T3N2M0 (13/38 positive nodes). Immunohistochemistry revealed cells positive for vimentin (Fig. 2) and epithelial membranous antigen (EMA), and negative for CK7, CD34, CD31, CD20, CD3, CD45, C-Kit, OCT-2. Immunostaining for integrase interactor 1 (INI-1) showed loss of nuclear expression, which is consistent with MERT. Fluorescence in situ hybridization (FISH) for the corresponding SMARCB1 was not performed in consideration of the immunohistochemical results. Our patient did not undergo adjuvant chemotherapy, and he is still alive and in good clinical conditions 48 months later, without recurrence (Fig. 1b).

\section{Literature review}

We performed a systematic review of the literature regarding cases of colonic intestinal MERT. Case reports and center series were identified by PubMed "MeSH" search up to October 2016. Search items included: MERT, malignant rhabdoid tumor, colon rhabdoid tumor, rhabdoid colon cancer, and rhabdoid carcinomas. The cases obtained were from 19 manuscripts plus our case report, for a total of 20 patients to be analyzed.
A thorough search of the literature revealed 19 cases of primary rhabdoid tumor of the colon. Table 1 shows the clinical and histological characteristics of all cases of poorly differentiated carcinoma of the colon with rhabdoid features found in the literature. This entity seems to be a disease of older people with a mean age of 68 years at presentation; only four recent articles described the disease in young patients ( $<50$ years). No gender predilection was evident (the male/female ratio was 10/9). The average size at presentation, which we calculated based on the tumor's longest diameter, was $8 \mathrm{~cm}$. The largest tumor was $15 \mathrm{~cm}$ and the smallest one was $4 \mathrm{~cm}$. These tumors were distributed equally along the colon. Only four patients did not have identifiable organ metastasis or positive nodes at presentation. Fourteen patients had at least one positive lymph node invasion or distant metastasis. From the 19 cases in the literature, 11 cases had mixed histologic characteristics with adenocarcinoma and rhabdoid features, whereas the remaining eight cases had "pure" rhabdoid features (Table 2).

All patients underwent surgical resection as the first line of therapy. The overall survival for this tumor, even after surgical intervention, seems to be limited, with a large majority of the patients surviving less than 6 months. Only three studies described a survival of 1 year; in these patients there was no distant metastatic organ disease at the time of presentation. Six patients received adjuvant chemotherapy, five of them were N1. One patient received 12 cycles of leucovorin, flurouracil, and oxaliplatin (FOLFOX) and he was still alive at the time the manuscript was written, surviving 36 months without recurrence or distant metastasis [4]. Another patient received a trial of capecitabine and oxaliplatin and had a 6-month overall survival [5]. A third patient received a trial of bevacizumab and cetuximab and had an 8-month survival [6]. A fourth patient received one cycle of 5-flurouracil, leucovorin, and oxaliplatin (mFOLFOX6), palliative radiosurgery to the rib, and three cycles of epirubicin, capecitabine and oxaliplatin

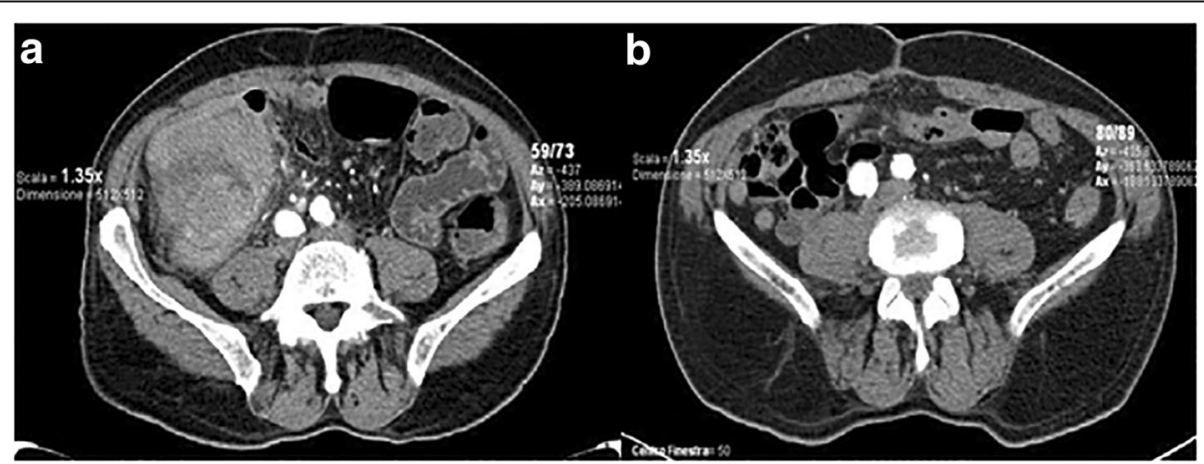

Fig. 1 Computed tomography scan describing a mass involving cecum and ascending colon (a) and the absence of recurrence at a 4-year follow-up (b) 


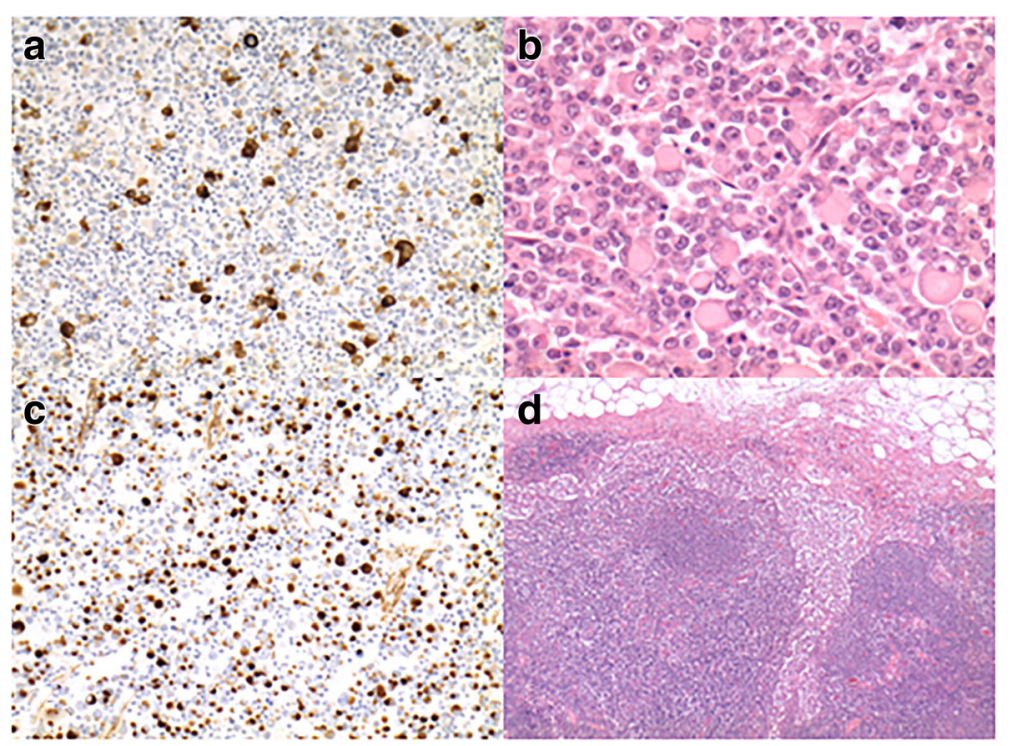

Fig. 2 Immunohistochemistry gross pathology of our case. a MNF 117 negative ( $\times 20)$; b hematoxylin and eosin tumor cells (c) rhabdoid component, and positive for vimentin $50-70 \%(\times 20)(\mathbf{d})$ positive node $(\times 5)$

(EOX), but she expired within 4 months of her diagnosis [7]. Samalavicius et al. treated their patient with FOLFOX4 and leucovorin, flurouracil, and irinotecan (FOLFIRI) regimen, with a survival of 7 months [8]. Han et al. described therapy with adjuvant radiotherapy (40 Gy) and chemotherapy (5-fluorouracil and oxaliplatin) [9].

\section{Discussion}

Malignant rhabdoid tumors (MRTs) are aggressive neoplasms that were first described in young children with renal primary neoplasms $[10,11]$. MRTs were later seen to arise at different locations, such as the central nervous system (CNS) [5], liver [6], genitourinary tract [7], and GI tract [8], known with the name of extrarenal rhabdoid tumors (MERTs). The histologic hallmark of MERTs is a centrically located and large nucleus, prominent nucleoli, abundant and eosinophilic cytoplasm, and paranuclear inclusions of intermediate filaments and abundant mitotic figures. Review of the pathologic characteristics of all cases available in the literature demonstrates that centric nuclei are the common feature among all MERTs.

These tumors are described as "composite" when the rhabdoid phenotype is mixed with another type of identifiable neoplasm and termed "pure" when the rhabdoid features are the only identifiable phenotype. When adenocarcinoma and rhabdoid tumor coexist, the rhabdoid features remained similar in all of them [4].

The histogenesis of MERT is unclear. Ota et al. [12] suggested that the rhabdoid tumor cells were derived from a primitive pluripotent cell that had the potential for a wide range of differentiation and accounted for the phenotypic heterogeneity observed in MERTs. In the literature review, we observed that the only patient alive in the "pure" subgroup had no involvement of lymphatic nodes or organ metastasis at the time of surgery, and underwent adjuvant chemotherapy and radiotherapy. In the "composite" subgroup, the patient with a longer survival had positive nodes and received chemotherapy (Table 2). This confirms the difficulty to define a prognosis based on histological findings and the absence of a standard therapeutic protocol, implying the relevance of performing an $\mathrm{R} 0$ resection in the first place.

The management of these tumors is usually surgical resection if the tumor is operable. The rare occurrence of MERT has made it complicated to establish adequate survival-improving protocols. Most of the reports indicate that MERTs are highly aggressive tumors, with more than $75 \%$ of patients dying within 6 months of the initial diagnosis $[3,4]$.

GI rhabdoid tumors are very rare. The majority of extrarenal rhabdoid tumors occur in children, while GI rhabdoid tumors are more common in the elderly population. Cytokeratin and vimentin are frequently found on immunochemistry.

Historically, all the rhabdoid colorectal cancers reported in the literature primarily affect older people, with ages ranging from 62 to 84 years of age and with no gender preference. Nevertheless, we found a few cases in young patients in our review.

Recent reviews suggested that these tumors are large and tend to be located in the transverse colon or 


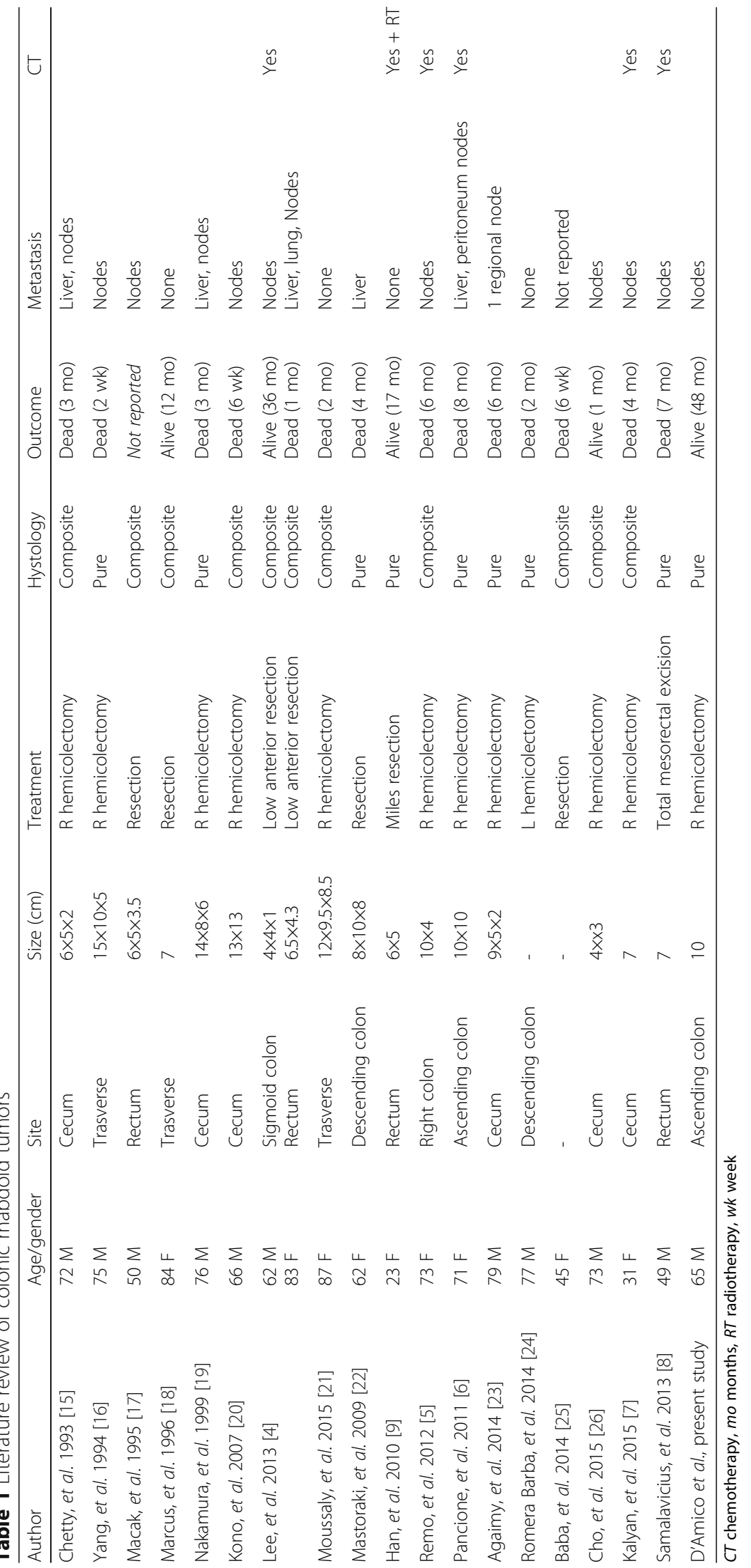


Table 2 Subgroup analysis (our case was excluded)

\begin{tabular}{lllllllll}
\hline & Cases & $\begin{array}{l}\text { Dead/max } \\
\text { survival }\end{array}$ & $\begin{array}{l}\text { Organ metastasis/ } \\
\text { nodes in dead }\end{array}$ & CT, RT in dead & $\begin{array}{l}\text { Alive/max } \\
\text { survival }\end{array}$ & $\begin{array}{l}\text { Organ metastasis/ } \\
\text { nodes in alive }\end{array}$ & CT, RT in alive & Survival literature review \\
\hline Pure & 8 & $7 / 7 \mathrm{mo}$ & $50 \% / 83 \%$ & $28,5 \%$ & $1 / 17 \mathrm{mo}$ & $0 \%, 0 \%$ & $100 \%$ & $12,5 \%$ \\
Composite & $11^{*}$ & $7 / 6 \mathrm{mo}$ & $33 \% / 83 \%$ & $28,5 \%$ & $3 / 36 \mathrm{mo}$ & $0 \%, 67 \%$ & $33,4 \%$ & $30 \%$ \\
\hline
\end{tabular}

$C T$ chemotherapy, mo months, $R T$ radiotherapy

*One patient did not have survival reported in the literature review

proximal ascending colon [3, 4], however, our literature review highlighted a predominance in cecum involvement. Surgical tumor resection was adopted by all series as first-line treatment, but despite it, survival remained low with a median survival of 7 months. We noted that patients alive at the time of publication were patients without metastasis at presentation or with limited node involvement. Nevertheless, two of four patients with no macroscopic metastasis or positive nodes during the surgical intervention, expired in less than 2 months. Horazdovsky et al. concluded in their meta-analysis that surgery and actiomycin therapy may improve survival in MERT [13]. Beneficial results of adjuvant treatment have been reported for MERTs in the skin and CNS, but not in the liver. Chemotherapy did not seem to impact survival (Table 1); also, Pancione et al. [6] and Remo et al. [5] reported that adjuvant chemotherapy had no significant benefit. The role of adjuvant treatment for MERT in the gastrointestinal tract is unknown. Since extrarenal rhabdoid tumors are rare, no standard therapeutic pathway exists and no randomized trials that examine the role of chemotherapy combinations or addition of new drugs have been done. Also, the use of radiotherapy did not improve survival in adults with MRTs [14].

We report an extraordinary case of a patient with long-term survival due to the extended surgical treatment. We believe that the absence of organ metastasis at the presentation should be a positive prognostic factor, although pathology confirmed node involvement (13/38 positive) on microscopy. Since we performed an extended right hemicolectomy with lymphadenectomy (upto the main nodes), omentectomy, and removal of the perirenal fat, we believe that the combination of no organ metastasis and careful extended surgery could explain this extraordinary case of long survival. Our Department of Oncology did not propose chemotherapy for our patient due to the absence of a therapeutic protocol with proven efficacy in the literature for this rare tumor. The follow-up CT scans showed absence of disease.

\section{Conclusions}

In conclusion, the current case report is, to our knowledge, the only case of a patient with colonic rhabdoid tumor with extended surgical resection with a survival of 4 years without recurrence or metastatic disease evidenced in control CT scans. All the published cases exhibit similar clinical-pathological features, as well as the characteristic histological features of sarcoma differentiation of rhabdoid cells, which appear to indicate aggressive biological behavior. Further investigations into this highly aggressive colonic carcinoma showing rhabdoid features are required in order to determine specific and effective treatment for this type of tumor.

\section{Abbreviations}

CK: Cytokeratin; CNS: Central nervous system; CT: Computed tomography; EGD: Esophagogastroduodenoscopy; EMA: Epithelial membranous antigen; FISH: Fluorescence in situ hybridization; Gl: Gastrointestinal; INI-1: Immunostaining for integrase interactor 1; MERT: Malignant extrarenal rhabdoid tumor; MRT: Malignant rhabdoid tumor

\section{Acknowledgements \\ Not applicable}

Funding

No grant supported this work

\section{Availability of data and materials}

The CT scan is saved in the e-health system of our hospital with the clinical history reported in the case report and is accessible any time if requested by the editor.

\section{Authors' contributions}

$F D^{\prime} A, A B$, and $M C$ contributed to study conception and design. $F D^{\prime} A, A B$, and $G C$ contributed to the acquisition of data. FD'A, $A B$, and $C M$ contributed to the analysis and interpretation of data. $A B, F D^{\prime} A, G G, C M$, and $G C$ contributed to the drafting of the manuscript. FD'A, MC, GG, and UC contributed to the critical revision of the manuscript. FD'A and AB performed the surgery. All authors read and approved the final manuscript.

Ethics approval and consent to participate

Not applicable

\section{Consent for publication}

Written informed consent was obtained from the patient for publication of this case report and any accompanying images. A copy of the written consent is available for review by the Editor-in-Chief of this journal.

\section{Competing interests}

The authors declare that they have no competing interests. All authors have agreed to the content of this manuscript and agree with its submission to the journal.

\section{Publisher's Note}

Springer Nature remains neutral with regard to jurisdictional claims in published maps and institutional affiliations.

\section{Author details}

'Department of Surgery, Oncology and Gastroenterology, Hepatobiliary Surgery and Liver Transplantation, Padova University, Padova, Italy. ${ }^{2}$ Department of Surgery, Division of Transplantation and Immunology, Yale University, New Haven, CT, USA. ${ }^{3}$ Department of Medicine-DIMED, Clinica dell'Ipertensione Arteriosa, University of Padova, Padova, Italy. ${ }^{4}$ Surgical 
Pathology and Cytopathology Unit, Department of Medicine (DIMED), University Hospital of Padova, Padova, Italy. ${ }^{5}$ Department of Surgery, Favaloro Foundation, Buenos Aires University, Buenos Aires, Argentina. ${ }^{6}$ Hepatobiliary Surgery and Liver Transplantation Unit, Department of Surgery, Oncology and Gastroenterology, University Hospital of Padova, Via Giustiniani 2, 35128 Padova, Italy.

Received: 29 April 2017 Accepted: 23 December 2017

Published online: 17 February 2018

\section{References}

1. Beckwith JB, Palmer NF. Histopathology and prognosis of Wilms tumors: results from the First National Wilms' Tumor Study. Cancer. 1978;41(5):193748. PMID:206343.

2. Malhotra Y, Fitzgerald TN, Jubinsky PT, Harper H, Silva CT, Zambrano E, et al. A unique case of rhabdoid tumor presenting as hemoperitoneum in an infant. J Pediatr Surg. 2011;46:247-51.

3. Toth L, Nemes Z, Gomba S, Asztalos L, Molnar C, Andras C, et al. Primary rhabdoid cancer of the ileum: a case report and review of the literature. Pathol Res Pract. 2010;206:110-5.

4. Lee SH, Seol H, Kim WY, Lim SD, Kim WS, Hwang TS, et al. Rhabdoid colorectal carcinomas: reports of two cases. Korean J Pathol. 2013;47:372-7.

5. Remo A, Zanella C, Molinari E, Talamini A, Tollini F, Piacentini P, et al. Rhabdoid carcinoma of the colon: a distinct entity with a very aggressive behavior: a case report associated with a polyposis coli and review of the literature. Int J Surg Pathol. 2012;20:185-90.

6. Pancione M, Di Blasi A, Sabatino L, Fucci A, Dalena AM, Palombi N, et al. A novel case of rhabdoid colon carcinoma associated with a positive CpG island methylator phenotype and BRAF mutation. Hum Pathol. 2011;42:1047-52.

7. Kalyan A, Pasricha G, Monga D, Singhi A, Bahary N. Case report of rhabdoid colon cancer and review of literature. Clin Colorectal Cancer. 2015;14:e5-8.

8. Samalavicius NE, Stulpinas R, Gasilionis V, Baltruskeviciene E, Aleknavicius E, Mickys U. Rhabdoid carcinoma of the rectum. Ann Coloproctol. 2013;29:252-5.

9. Han SL, Li JL, Liu Z, Cheng J, Guo SC, Wu SL. Malignant rhabdoid tumor of rectum: report of a case. Tech Coloproctol. 2010;14:199-200.

10. Haas JE, Palmer NF, Weinberg AG, Beckwith JB. Ultrastructure of malignant rhabdoid tumor of the kidney. A distinctive renal tumor of children. Hum Pathol. 1981;12:646-57.

11. Palmer NF, Sutow W. Clinical aspects of the rhabdoid tumor of the kidney: a report of the National Wilms' Tumor Study Group. Med Pediatr Oncol. 1983; 11:242-5.

12. Ota S, Crabbe DC, Tran TN, Triche TJ, Shimada H. Malignant rhabdoid tumor. A study with two established cell lines. Cancer. 1993;71:2862-72.

13. Horazdovsky R, Manivel JC, Cheng EY. Surgery and actinomycin improve survival in malignant rhabdoid tumor. Sarcoma. 2013;2013:315170.

14. Sultan I, Qaddoumi I, Rodriguez-Galindo C, Nassan AA, Ghandour K, AlHussaini M. Age, stage, and radiotherapy, but not primary tumor site, affects the outcome of patients with malignant rhabdoid tumors. Pediatr Blood Cancer. 2010:54:35-40.

15. Chetty R, Bhathal PS. Caecal adenocarcinoma with rhabdoid phenotype: an immunohistochemical and ultrastructural analysis. Virchows Arch A Pathol Anat Histopathol. 1993;422:179-82.

16. Yang $\mathrm{AH}$, Chen WY, Chiang $\mathrm{H}$. Malignant rhabdoid tumour of colon. Histopathology. 1994;24:89-91.

17. Macak J, Kodet R. Rectal adenocarcinoma with rhabdoid phenotype. Pathologica. 1995;87:696-9.

18. Marcus VA, Viloria J, Owen D, Tsao MS. Malignant rhabdoid tumor of the colon. Report of a case with molecular analysis. Dis Colon Rectum. 1996;39: 1322-6.

19. Nakamura I, Nakano K, Nakayama K, Ishii Y, Ohta K, Takahashi M, et al. Malignant rhabdoid tumor of the colon: report of a case. Surg Today. 1999; 29:1083-7.

20. Kono T, Imai Y, Imura J, Ono Y, Hagiwara S, Taira K, et al. Cecal adenocarcinoma with prominent rhabdoid feature: report of a case with immunohistochemical, ultrastructural, and molecular analyses. Int J Surg Pathol. 2007:15:414-20.

21. Moussaly E, Atallah JP. A rare case of undifferentiated carcinoma of the colon with rhabdoid features: a case report and review of the literature. Case Rep Oncol Med. 2015;2015:531348.
22. Mastoraki A, Kotsilianou O, Papanikolaou IS, Foukas PG, Sakorafas G, Safioleas M. Malignant rhabdoid tumor of the large intestine. Int J Colorectal Dis. 2009;24:1357-8.

23. Agaimy A, Rau TT, Hartmann A, Stoehr R. SMARCB1 (INI1)-negative rhabdoid carcinomas of the gastrointestinal tract: clinicopathologic and molecular study of a highly aggressive variant with literature review. Am J Surg Pathol. 2014;38:910-20

24. Romera Barba E, Sanchez Perez A, Duque Perez C, Garcia Marcilla JA, Vazquez Rojas JL. Malignant rhabdoid tumor of the colon: a case report. Cir Esp. 2014;92:638-40.

25. Baba Y, Uchiyama T, Hamada K, Ishihara Y, Tanaka H, Isono Y, et al. A case report of undifferentiated carcinoma of the sigmoid colon with rhabdoid features. Nihon Shokakibyo Gakkai Zasshi. 2014;111:1384-90.

26. Cho IJ, Kim SS, Min YD, Noh MW, Hong R. Poorly differentiated cecal adenocarcinoma showing prominent rhabdoid feature combined with appendiceal mucinous cystadenoma: a case report and review of the literature. Oncol Lett. 2015;9:1527-30.

\section{Submit your next manuscript to BioMed Central and we will help you at every step:}

- We accept pre-submission inquiries

- Our selector tool helps you to find the most relevant journal

- We provide round the clock customer support

- Convenient online submission

- Thorough peer review

- Inclusion in PubMed and all major indexing services

- Maximum visibility for your research

Submit your manuscript at www.biomedcentral.com/submit
Biomed Central 